\title{
CUT POINTS OF CONNECTED SETS AND OF CONTINUA*
}

\author{
BY \\ G. T. WHYBURN
}

1. Introduction. In this paper it will be shown, among other things, that the set $G$ of all the cut points of any continuum $M$ in a locally compact, metric, and separable space is a $G_{\delta \sigma}$, i.e., the sum of a countable number of $G_{b}$ sets. $\dagger$ From this result, with the aid of a well known theorem of W. H. Young's, it follows that if the set $G$ is uncountable it must contain a perfect set. $\ddagger$ Thus the set of all cut points of any continuum is either vacuous, finite, countable, or of the power of the continuum.

The customary notation and terminology of point set theory will be employed. For example, $\bar{X}=X+X^{\prime}$, where $X^{\prime}$ is the set of all limit points of the set $X ; K \subset B$ means that $K$ is a subset of $B$ or that $B$ contains $K$; $K \cdot H$ denotes the set of points common to $K$ and $B ; \rho(X, Y)$ denotes the distance from $X$ to $Y$ when $X$ and $Y$ are points, and denotes the minimum distance between $X$ and $Y$ when $X$ and $Y$ or either contains more than one point, i.e. the greatest lower bound of the aggregate of numbers $[\rho(x, y)]$, where $x$ and $y$ are points of $X$ and $Y$ respectively; and $\delta(M)$ denotes the diameter of the set $M$, i.e., the least upper bound of the aggregate [ $\rho(x, y)]$, where $x$ and $y$ are two points of $M$. In addition, if $X$ is a cutting of a connected set $M$, unless otherwise stated, the equation $M-X=M_{a}(X)+$ $M_{b}(X)$ is to be interpreted as meaning that $M-X$ is the sum of the two mutually separated sets $M_{a}(X)$ and $M_{b}(X)$ containing the sets $A$ and $B$ respectively. When this equation is true, $X$ is said to separate $A$ and $B$ in $M$.

All point sets considered are assumed to lie in a locally compact, metric, and separable space.

2. Cut points and regular points. We prove the following theorem:

* Presented to the Society, December 27, 1928. Received by the editor of the Bulletin in December, 1928, accepted for publication in the Bulletin, and subsequently transferred to the Transactions.

$\dagger$ It has been shown by C. Zarankiewicz that the set of all cut points of any continuous curve is an $F_{\sigma}$ but that this is not true of continua in general; see Sur les points de division dans les ensembles connexes, Fundamenta Mathematicae, vol. 9 (1927), pp. 124-171; see pp. 163, 164.

$\ddagger$ According to results due to F. Bernstein (Leipziger Berichte, vol. 60 (1908), p. 325), there exist uncountable sets, even uncountable linear sets, which contain no perfect set. The theorems in this paper show, however, that such a set cannot be the set of cut points of any continuum. 
Theorem 1. If $A$ and $B$ are any two mutually exclusive closed subsets of a connected set $M$ and $K$ is the set of all those points of $M$ which separate $A$ and $B$ in $M$, then $K+A+B$ contains every point $P$ of $\bar{K}$ which is a regular point* of $M+P$.

Let $P$ be any limit point of $K$ which is not in $A+B$ and which is a regular point of $M+P$. As shown in my paper Concerning connected and regular point sets $\dagger$ either there exists a sequence $X_{1}, X_{2}, X_{3}, \cdots$ of points of $K$ having $P$ as its sequential limit point and such that, for every positive integer $i$,

$$
M-X_{i}=M_{a}\left(X_{i}\right)+M_{b}\left(X_{i}\right), \sum_{n=1}^{i-1} X_{n} \subset M_{a}\left(X_{i}\right), \text { and } \sum_{n=i+1}^{\infty} X_{n} \subset M_{b}\left(X_{i}\right),
$$

or such a sequence exists such that, for each $i$,

$$
\sum_{n=1}^{i-1} X_{n} \subset M_{b}\left(X_{i}\right) \text { and } \sum_{n=i+1}^{\infty} X_{n} \subset M_{a}\left(X_{i}\right) \text {. }
$$

The two cases are alike so we shall consider only the former. Let $E=$ $\sum M_{a}\left(X_{i}\right)$, and let $F=M-E$. Then $F=\Pi M_{b}\left(X_{i}\right)$, i.e. $F$ is identically the set of points common to all the sets $\left[M_{b}\left(X_{i}\right)\right]$. Thus no point of $E$ is a limit point of $F$, because each point of $E$ belongs to a set $M_{a}\left(X_{k}\right)$, and $F \subset M_{b}\left(X_{k}\right)$ for every $k$. I shall now show that no point of $F$ except possibly $P$ (in case $P$ belongs to $M$ ) is a limit point of $E$. Suppose, on the contrary, that such a limit point $Q$ of $E$ does exist. Let $\epsilon$ be a positive number which is $<\rho(P, Q+A+B)$. Since $P$ is a regular point of $M+P$, there exists a finite subset $U$ of $M$ which $\epsilon$-separates $P$ in $M+P$, i.e., $(M+P)-U=$ $M_{p}+M_{0}$, where $M_{p}$ and $M_{0}$ are separated, $M_{p} \supset P$, and $\delta\left(M_{p}\right)<\epsilon$. Now $M_{0}+U$ is the sum of a finite number of mutually separated connected point

* The point $P$ of a connected point set $M$ is called a Menger regular point of $M$, or simply a regular point of $M$, if for each $\epsilon>0, P$ can be $\epsilon$ separated in $M$ by some finite subset $U$ of $M$, i.e., a finite subset $U$ of $M$ exists such that $M-U=M_{p}(U)+M(U)$, where $M_{p}(U)$ and $M(U)$ are mutually separated, $M_{p}(U) \supset P$, and $\delta\left[M_{p}(U)\right]<\epsilon$. If the $\epsilon$-separating set $U$ can, for every $\epsilon>0$, be chosen of power $\leqq n$ but cannot, for every $\epsilon$, be chosen of power $<n$, then $P$ is a point of order $n$ of $M$. See K. Menger, Grundzüge einer Theorie der Kurven, Mathematische Annalen, vol. 95 (1925), pp. 277306, and P. Urysohn, Sur la ramification des lignes Cantoriennes, Comptes Rendus, vol. 175 (1922), p. 481.

† Bulletin of the American Mathematical Society, vol. 33 (1927), pp. 685-689, see proof of Theorem 1. The proof here given is somewhat similar to the one just referred to. R. L. Wilder has recently published some interesting extensions of Theorem 1 in my paper here cited; see R. L. Wilder, On connected and regular point sets, Bulletin of the American Mathematical Society, vol. 34 (1928), pp. 649-655.

† See Knaster and Kuratowski, Remark on a theorem of $R$. L. Moore, Proceedings of the National Academy of Sciences, vol. 13 (1927), pp. 647-649; see also an abstract of mine in the Bulletin of the American Mathematical Society, vol. 33 (1927), p. 388. 
sets $E_{1}, E_{2}, E_{3}, \cdots, E_{n}$. One of these, say $E_{k}$, contains $Q$, and $Q$ is not a limit point of $M-E_{k}$. But there exists an integer $m$ such that for every integer $j>m, X_{j} \subset M_{p}$. And since $Q \subset M_{b}\left(X_{j}\right), E_{k} \supset Q$, and $E_{k} \cdot X_{j}=0$, then $E_{k} \subset M_{b}\left(X_{j}\right)$ for every $j>m$. Hence $Q$ is not a limit point of $\sum_{j-m+1}^{\infty} M_{a}\left(X_{j}\right)$, because this set of points is a subset of $M-E_{k}$. But clearly $Q$ is not a limit point of $\sum_{j=1}^{m} M_{a}\left(X_{j}\right)$, because $Q \subset F \subset M_{b}\left(X_{j}\right)$ for every $j$. Therefore $Q$ is not a limit point of $E$, contrary to supposition. Thus no point of $F$ except possibly $P$ can be a limit point of $E$.

Now $P$ must belong to $F$, for if not, then $E$ and $F$ are mutually separated; and since $E+F=M$, this contradicts the fact that $M$ is connected. Hence $P \subset F$; and since $E$ and $F-P$ are mutually separated and contain $A$ and $B$ respectively, and $E+(F-P)=M-P$, therefore $P$ separates $A$ and $B$ in $M$ and hence belongs to $K$.

Corollary 1a. If $M$ is a continuum, then every point of $\bar{K}$ which is not in $K+A+B$ is a non-regular point of $M$.

Theorem 1 does not hold true, even in case $M$ is a continuum, when we substitute the words "point of connectivity im kleinen" for the words "regular point." For let $I$ be the interval $(0,2)$ of the $X$-axis; let $A$ and $B$ be the end points of $I$; let $L$ be the straight line interval from $(1,0)$ to $(1,1)$; let $P$ be the point $(1,0)$; for each positive or negative integer $n$, let $L_{n}$ be the straight line interval from $(1+1 /(2 n), 0)$ to $(1+1 /(2 n), 1)$; and let $M$ be the continuum $I+L+\sum L_{n}$. Then the set $K$ of points of $M$ which separate $A$ and $B$ in $M$ is identical with the set $I-(A+B+P)$; and $P$ is a limit point of $K$ and is a point of connectivity im kleinen of $M$; but $P$ does not belong to $K$.

THEOREM 2. Let $N$ be any closed subset of a connected point set $M$, and let $K$ be the set of all points of $M$ which separate* $N$ in $M$. Then $K+N$ contains every point $P$ of $\bar{K}$ which is a regular point of $M+P$.

Let $P$ be any point of $\bar{K}$ which is not in $N$ and which is a regular point of $M+P$, let $\epsilon$ be a positive number which is $<\rho(P, N)$, and let $U$ be a finite subset of $M$ which $\epsilon$-separates $P$ in $M+P$. Then $M-U=M_{p}+M_{n}$, where $M_{p}$ and $M_{n}$ are separated and contain $P$ and $N$ respectively. Now $M_{p}$ contains an infinite sequence $X_{1}, X_{2}, X_{3}, \cdots$ of points of $K$ having $P$ for its sequential limit point. For each $i, M-X_{i}=M_{1}\left(X_{i}\right)+M_{2}\left(X_{i}\right)$, where $M_{1}\left(X_{i}\right)$ and $M_{2}\left(X_{i}\right)$ are separated and $N \cdot M_{1}\left(X_{i}\right) \neq 0 \neq N \cdot M_{2}\left(X_{i}\right)$. Now since $\dagger$ $M_{n}+U$ is the sum of a finite number of connected point sets, it follows that there exist two point sets $K_{a}$ and $K_{b}$ and an infinite sliosequence

* The point $P$ of a connected set $M$ is said to separate a given subset $N$ of $M$ in $M$ if $M-P$ is separated between some two points of $N$, i.e., $M-P$ is the sum of two mutually separated sets $M_{1}$ and $M_{2}$, where $N \cdot M_{1} \neq 0 \neq N \cdot M_{2} ;$ see R. L. Wilder, loc. cit.

† See the reference to Knaster and Kuratowski above. 
$X_{n_{1}}, X_{n_{2}}, \cdots$ of the sequence $\left[X_{n}\right]$ such that $K_{a}+K_{b}=M_{n}+U$ and such that, for every $i, K_{a} \subset M_{1}\left(X_{n_{i}}\right)$ and $K_{b} \subset M_{2}\left(X_{n_{i}}\right)$. Hence if $A=N \cdot K_{a}$ and $B=N \cdot K_{b}$, then $A$ and $B$ are mutually exclusive, closed, and nonvacuous subsets of $M$, and every point of $\sum X_{n i}$ separates $A$ and $B$ in $M$. Then since $P$ is a limit point of $\sum X_{n i}$ and is a regular point of $M+P$, it follows by Theorem 1 that $P$ belongs to $M$ and separates $A$ and $B$ in $M$. Therefore $P$ separates $N$ in $M$ and hence belongs to $K$. This completes the proof.

Theorem 3. Let $A$ and $B$ be any two mutually exclusive closed subsets of a continuum $M$, and let $K$ be the set of all those points of $M$ which separate $A$ and $B$ in $M$. Then $K+A+B$ contains every point $P$ of $\bar{K}$ having the property that every subcontinuum of $M$ containing $P$ contains at least two points at which $M$ is connected im kleinen.

Let $P$ be a point of $\bar{K}$ not in $A+B$ which has the property mentioned in this theorem. Let the points and point sets $X_{1}, X_{2}, X_{3}, \cdots, E$, and $F$ be selected and defined exactly as in the proof of Theorem 1. Then, just as in that proof, no point of $E$ is a limit point of $F$. Likewise no point of $F-P$ can be limit point of $E$. For suppose some point $Q$ of $F-P$ is a limit point of $E$. Let $R$ be a compact neighborhood of $P$ such that $\bar{R}$ does not contain $Q$. Then since for no $i$ can $Q$ be a limit point of $M_{a}\left(X_{i}\right)$, it readily follows that if, for each $i, I\left(X_{i}, X_{i+1}\right)$ denotes the set of points $M_{b}\left(X_{i}\right) \cdot M_{a}\left(X_{i+1}\right)+$ $X_{i}+X_{i+1}$, then both $Q$ and $P$ belong to the sequential limiting set $L$ of the sequence of continua* $\left[I\left(X_{i}, X_{i+1}\right)\right]$. Now with the aid of a theorem of Janiszewski's $\dagger$ and a theorem of Lubben's, $\ddagger$ it follows that $L \cdot \bar{R}$ contains a continuum $H$ containing $P$ and at least one point of the boundary of $R$. Since for each $i, L \cdot M_{a}\left(X_{i}\right)=0$, clearly $H \subset F$. Now by hypothesis, $H$ contains a point $C$, distinct from $P$, at which $M$ is connected im kleinen. Since $C$ belongs to $L$, it follows that there exists an integer $k$ and a point $D$ of $I\left(X_{k}, X_{k+1}\right)$ which can be joined to $C$ by a subcontinuum $I$ of $M$ which does not contain $P$. There exists an integer $j>k$ such that $X_{j}$ does not belong to $I$. But then $C$ belongs to $M_{b}\left(X_{j}\right)$ (for $C \subset F \subset M_{b}\left(X_{i}\right)$, for every $i$ ), $D$ belongs to $M_{a}\left(X_{j}\right)$, and $I$ is a connected subset of $M_{a}\left(X_{j}\right)+M_{b}\left(X_{j}\right)$ containing both $C$ and $D$. Clearly this is impossible, because $M_{a}\left(X_{j}\right)$ and $M_{b}\left(X_{i}\right)$

* See my paper Concerning the cut points of continua, these Transactions, vol. 30 (1928), pp. 597609, Theorem 1.

$\dagger$ Z. Janiszewski, Sur les continus irréductibles entre deux points, Journal de l'Ecole Polytechnique, (2), vol. 16 (1912), p. 109.

$\ddagger$ R. G. Lubben, Concerning limiting sets in abstract spaces, these Transactions, vol. 30 (1928), pp. 668-685; see Theorems 11 and 12. 
are mutually separated. Thus the supposition that $F-P$ contains a limit point of $E$ leads to a contradiction. Hence $E$ and $F-P$ are mutually sepparated; and since $E \supset A, F-P \supset B$, and $E+(F-P)=M-P$, it follows that $P$ separates $A$ and $B$ in $M$ and hence belongs to $K$.

3. Cardinal number and Borel classification of the set of cut points. We shall prove the following theorem:

Theorem 4. Let $A$ and $B$ be any two points, or, indeed, any two closed mutually exclusive subsets, of a continuum $M$, and let $K$ be the set of all points of $M$ which separate $A$ and $B$ in $M$. Then $K$ is the sum of $a G_{b}$ set* and a countble set.

Let $E$ be the set of all those points of $M$ which can, for each $\epsilon>0$, be $\epsilon$-separated in $M$ by some two points of $K$, i.e., the set of all points of $M$ of order $\leqq 2$ relative to $K$. It follows readily with the aid of a theorem of Menger's $\dagger$ that if $E$ exists it is a $G_{\delta}$ set. And since $A+B$ is closed, $H=E-$ $E \cdot(A+B)$ is a $G_{\delta}$ set. Now since every point of $E$ is a limit point of $K$ and is a regular point of $M$, then by Theorem $1, K+A+B \supset E$. Hence $B \subset K$. And by a theorem of the author's, $\ddagger K-H$ is countable. This completes the proof.

Corollary $\$ 4 \mathrm{a}$. If $K$ is uncountable, it contains a perfect set.

THEOREM 5. The set $G$ of all the cut points of any continuum $M$ is a $G_{80}$, i.e., the sum of a countable number of $G_{\delta}$ sets.

Since our space is metric and separable, $M$ itself is $\|$ separable and hence contains a countable set $D$ such that $\dot{M}=\bar{D}$. Let $H$ be the collection of all possible pairs of points of $D$, and for each pair $(A, B)$ of points in $H$, let $K_{a b}$ be the set of all points of $M$ which separate $A$ and $B$ in $M$. By Theorem 4, $K_{a b}=\mathrm{a} G_{b}$ set + a countable set, for every pair $(A, B)$ in $H$. Then since $\boldsymbol{H}$ is countable, it follows that $\sum_{H} K_{a b}$ is a $G_{\delta \sigma}$. Now if $X$ is any point of $G, M-X=M_{1}+M_{2}$, where $M_{1}$ and $M_{2}$ are mutually separated. And if $P_{1}$ and $P_{2}$ are points of $M_{1}$ and $M_{2}$, respectively, then since $P_{1} \cdot M_{2}^{\prime}=0=P_{2} \cdot M_{1}^{\prime}$,

${ }^{*} \mathrm{~A} G_{\delta}$ set is a point set which is the common part of some family of open sets.

$\dagger$ See K. Menger, loc. cit., Theorem 3.

¥ See Concerning the cut points of continua, loc. cit., Theorem 7; see also my paper Concerning collections of cuttings of connected point sets, Bulletin of the American Mathematical Society, vol. 35 (1929), pp. 87-104, Theorem 12.

\$ This corollary follows from Theorem 4 and Young's theorem that every $G_{\diamond}$ set containing a subset which is dense in itself contains a perfect set; see W. H. Young, Leipziger Berichte, vol. 55 (1903), p. 287.

\| See W. Gross, Zur Theorie der Mengen, in denen ein Distanzbegriff definiert ist, Wiener Sitzungsberichte, vol. 123 (1924), pp. 801-819. 
and $P_{1}+P_{2} \subset \bar{D}$, then $D$ is a subset of neither $M_{1}+X$ nor $M_{2}+X$. Hence there exist points $A$ and $B$ of $D$ in $M_{1}$ and $M_{2}$ respectively. Therefore $X$ separates $A$ and $B$ in $M$ and hence belongs to $K_{a b}$ and to $\sum K_{a b}$. Thus $G \equiv \sum K_{a b}$, and hence $G$ is a $G_{\delta \sigma}$.

Corollary* 5a. If $G$ is uncountable, it contains a perfect set.

We note here the following fact which, in a certain sense, is a converse proposition to Theorem 4:

If $K$ is any set which lies within the linear interval $I(A, B)$ and is the sum of $a G_{b}$ set and a countable set, then there exists a continuum $M$ such that $K$ is identically the set of all those points of $M$ separating $A$ and $B$ in $M$.

We note also the fact that the set of all the cut points of a continuum is not necessarily the sum of a $\mathrm{G}_{\delta}$ set and a countable set. Indeed, the set of all cut points of the universal acyclic continuous curve of Wazewski is not the sum of $a G_{\delta}$ set and a countable set.

4. Concluding remarks. Theorem 5 and Corollary $5 \mathrm{a}$ are propositions concerning the set $G$ of all the cut points of a continuum $M$. We may obtain the same conclusions for the set $H$ of all the local separating points $\dagger$ of a continuum $M$. We state the following propositions concerning the local separating points of continua.

(i) Every continuum $M$ contains a countable collection $\left[M_{i}\right](i=1,2,3$, ...) of compact subcontinua such that if $H$ is the set of all local separating points of $M$ and, for each $i, G_{i}$ is the set of all cut points of $M_{i}$, then (1) $\sum_{1}^{\infty} G_{i} \subset H$, (2) $H-\sum G_{i}$ is countable, and (3) for each $i, M_{i} \cdot\left(M-M_{i}\right)$ contains exactly two points.

(ii) The set $H$ of all the local separating points of any continuum $M$ is a $G_{\delta \sigma}$; and if $H$ is uncountable, it contains a perfect set.

(iii) Every continuous curve $M$ contains a countable collection $\left[M_{i}\right]$, $(i=1,2,3, \cdots)$ of compact continuous curves such that if $H$ is the set of all

* This corollary can be deduced from Theorem 5 using Young's theorem (loc. cit.), or it follows also from Corollary $4 \mathrm{a}$ and the author's theorem (see Concerning the cut points of continua, loc. cit., Theorem 3) that if $G$ is any uncountable set of cut points of $M$, then some two points $A$ and $B$ of $G$ are separated in $M$ by uncountably many points of $G$.

$\dagger$ The point $P$ of a continuum $M$ is a local separating point of $M$ if there exists a compact neighborhood $R$ of $P$ such that $M \cdot \bar{R}-P$ is separated between some two points of the component of $M \cdot \bar{R}$ which contains $P$; see my paper Local separating points of continua, in Monatshefte für Mathematik und Physik, vol. 35, No. 2. 
local separating points of $M$ and, for each $i, G_{i}$ is the set of all cut points of $M_{i}$, then $\sum_{1}^{\infty} G_{i} \equiv H$.

Result (i) is readily deduced from the theorems in my paper Local separating points of continua (loc. cit.)-in particular from the result that all save possibly a countable number of the local separating points of any continuum $M$ are points of order two of $M$. Result (ii) follows immediately from (i) and Theorem 5 and Corollary 5a above. Result (iii) was established incidentally by the author in proving the theorem* that the set $H$ of all the local separating points of any continuous curve is an $F_{\sigma}$.

It has been shown by the author $\dagger$ that all save possibly a countable number of the cut points of any continuum $M$ are points of order two of $M$. That this theorem does not hold true for connected sets $M$, even in case $M$ is irreducibly connected between some two of its points, is shown in the example given by Vietoris $\ddagger$ of a totally discontinuous function $y=\omega(x)$ which has a connected graph. That this theorem does not hold true even for connected and connected im kleinen sets $M$ is shown by the following example. Let $K$ be a non-dense perfect set on the interval $I(0,1)$ of the $X$-axis not containing the end points $A$ and $B$ of $I$. Let $S_{1}, S_{2}, S_{3}, \cdots$ be the complementary segments of $K$ in $I$. For each $i$, let $E_{i}$ be an ellipse with minor axis $\bar{S}_{i}$ and with major axis two units in length. Let $M=I+\sum_{1}^{\infty} E_{i}$. Then $M$ is connected and connected im kleinen, and every point of $K$ separates $A$ and $B$ in $M$; but clearly no point of $K$ is a regular point of $M$.

Recently I have made a study of the structure of connected and connected im kleinen point sets and, among other results, have found the following theorem:

In a connected and connected im kleinen point set $M$, every connected set of cut points is arcwise connected.§

\footnotetext{
* See my paper Concerning points of continuous curves defined by certain im kleinen properties Mathematische Annalen, vol. 102 (1929), pp. 133-336.

† See Concerning the cut points of continua, loc. cit., Theorem 7.

$\ddagger$ L. Vietoris, Stetige Mengen, Monatshefte für Mathematik und Physik, vol. 31 (1921), p. 202; see also Knaster and Kuratowski, Sur quelques propriétés topologiques des fonctions dérivées, Rendiconti del Circolo Matematico di Palermo, vol. 49 (1925).

$\S$ For special cases of this theorem, see an abstract by C. M. Cleveland in the Bulletin of the American Mathematical Society, vol. 32 (1926), p. 420, where the theorem is stated for plane continuous curves $M$, and my paper Concerning the structure of a continuous curve, American Journal of Mathematics, vol. 50 (1928), pp. 167-194, Theorem 8, where the theorem, in more general form, is proved for continuous curves $M$ in $n$-dimensional space.
} 
It is of interest in this connection to note the theorem, easily deducible from the work of F. Frankl ${ }^{*}$, that Every connected point set $M$ each point of which is of order $\leqq 2$ of $M$ is arcwise connected.

* See Ueber die zusammenhïngenden Mengen von höchstens zweiter Ordnung, Fundamenta Mathematicae, vol. 11 (1927), pp. 96-104.

UNIVERSTTY OF TEXAS,

AUstrin, Texas 